\title{
Glycodiversification for the Optimization of the Kanamycin Class Aminoglycosides
}

\author{
Jinhua Wang, Jie Li, Hsiao-Nung Chen, Huiwen Chang, Christabel Tomla Tanifum, \\ Przemyslaw G. Czyryca, Hsiu-Hsiang Liu, Cheng-Wei Tom Chang*
}

Department of Chemistry and Biochemistry, Utah State University, 0300 Old Main Hill, Logan, Utah 84322-0300, U.S.A.

Tel: +4357973545. Fax: +4357973390. Email: chang@cc.usu.edu

\section{Supporting Information}

HPLC and mass spectrometric analysis of Kanamycin B Analogs (S2-S25) 


\section{HPLC and mass spectrometric analysis of Kanamycin B Analogs}

HPLC analysis of the synthesized kanamycin B analogs was performed by using the Dionex HPLC system equipped with CarboPac analytical column. The samples were eluted with $5 \mathrm{mM}$ or $10 \mathrm{mM} \mathrm{KOH}$ solution. Few samples that show multiple components are not the result of impurities. It is likely due to the presence of different protonated stage of compounds, which is common for poly-cationic compounds like aminoglycosides.

\begin{tabular}{|l|c|}
\hline \multicolumn{1}{|c|}{ entry } & page \\
\hline HPLC profile and mass spec analysis for $\mathbf{1 6}$ & $\mathrm{S} 3-\mathrm{S} 4$ \\
\hline HPLC profile and mass spec analysis for $\mathbf{1 7}$ & $\mathrm{S} 4-\mathrm{S} 5$ \\
\hline HPLC profile and mass spec analysis for $\mathbf{1 8}$ & $\mathrm{S} 6-\mathrm{S} 7$ \\
\hline HPLC profile and mass spec analysis for $\mathbf{1 9}$ & $\mathrm{S} 7-\mathrm{S} 8$ \\
\hline HPLC profile and mass spec analysis for $\mathbf{2 0}$ & $\mathrm{S} 9-\mathrm{S} 10$ \\
\hline HPLC profile and mass spec analysis for $\mathbf{2 1}$ & $\mathrm{S} 10-\mathrm{S} 11$ \\
\hline HPLC profile and mass spec analysis for $\mathbf{2 2}$ & $\mathrm{S} 12-\mathrm{S} 13$ \\
\hline HPLC profile and mass spec analysis for $\mathbf{2 3}$ & $\mathrm{S} 13-\mathrm{S} 14$ \\
\hline HPLC profile and mass spec analysis for $\mathbf{7 7}$ & $\mathrm{S} 15-\mathrm{S} 16$ \\
\hline HPLC profile and mass spec analysis for $\mathbf{7 8}$ & $\mathrm{S} 16-\mathrm{S} 17$ \\
\hline HPLC profile and mass spec analysis for $\mathbf{7 9}$ & $\mathrm{S} 18-\mathrm{S} 19$ \\
\hline HPLC profile and mass spec analysis for $\mathbf{8 0}$ & $\mathrm{S} 19-\mathrm{S} 20$ \\
\hline HPLC profile and mass spec analysis for $\mathbf{8 1}$ & $\mathrm{S} 21-\mathrm{S} 22$ \\
\hline HPLC profile and mass spec analysis for $\mathbf{8 2}$ & $\mathrm{S} 22-\mathrm{S} 23$ \\
\hline HPLC profile and mass spec analysis for $\mathbf{8 3}$ & $\mathrm{S} 24-\mathrm{S} 25$ \\
\hline
\end{tabular}




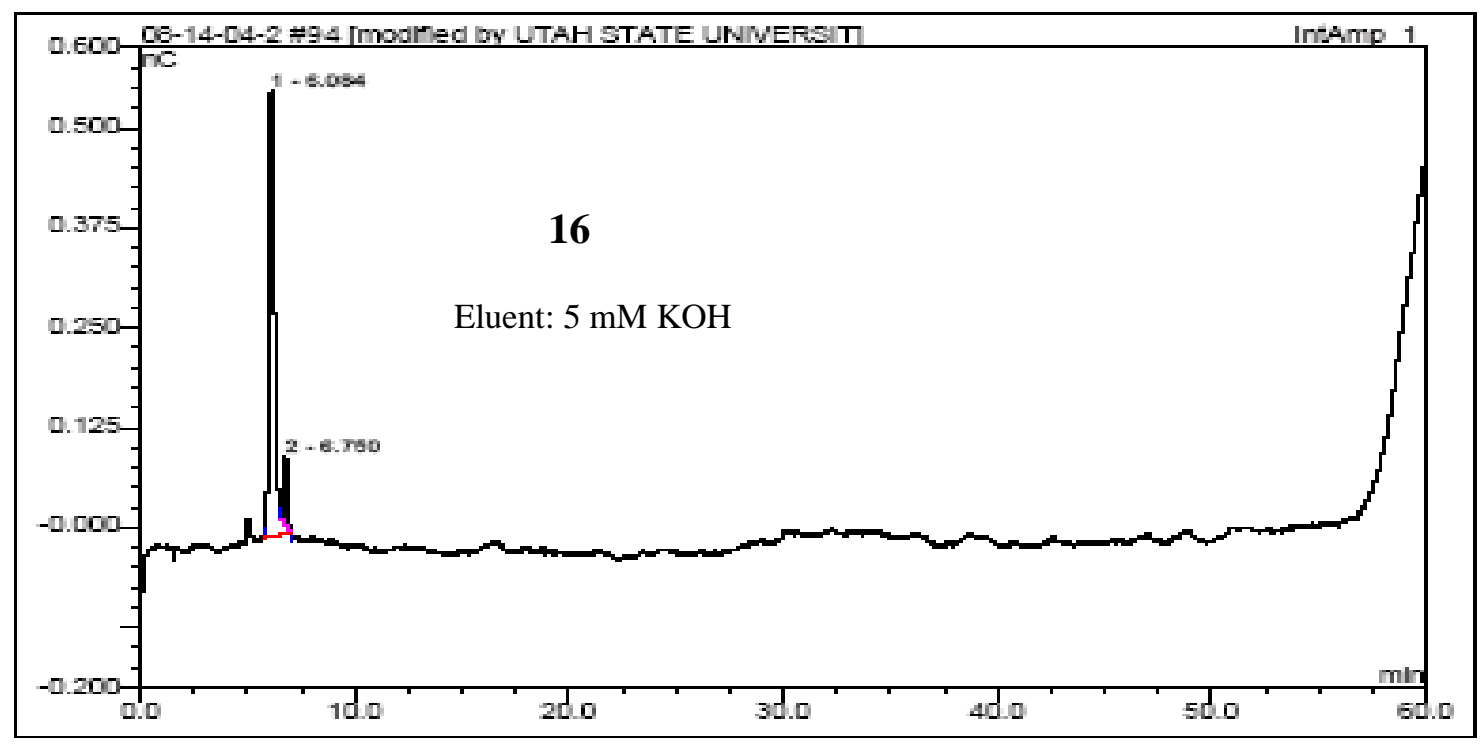

\begin{tabular}{|c|c|c|c|c|c|c|c|}
\hline No. & $\begin{array}{c}\text { Ret.TIme } \\
\mathrm{m} / \mathrm{n}\end{array}$ & Peak Name & $\begin{array}{c}\text { Helght } \\
\text { nc }\end{array}$ & $\begin{array}{c}\text { Area } \\
\text { nc*min }\end{array}$ & $\begin{array}{c}\text { Rel.Area } \\
\%\end{array}$ & Amount & Type \\
\hline 1 & 6.08 & n.a. & 0.558 & 0.185 & 92.33 & n.a. & BMS \\
\hline 2 & 6.75 & n.a. & 0.084 & 0.015 & 7.67 & n.a. & $R d$ \\
\hline Total: & & & 0.642 & 0.201 & 100.00 & 0.000 & \\
\hline
\end{tabular}

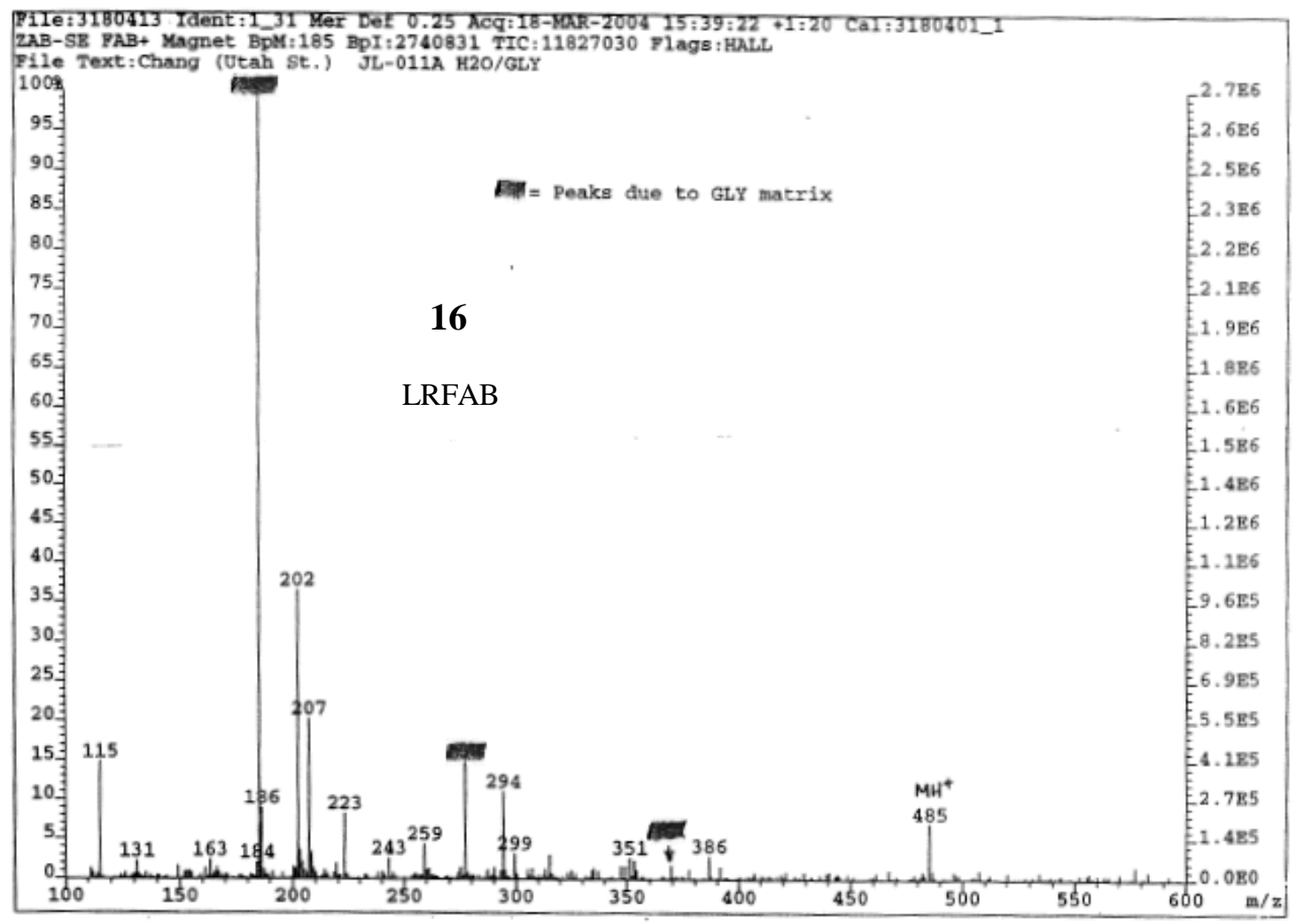



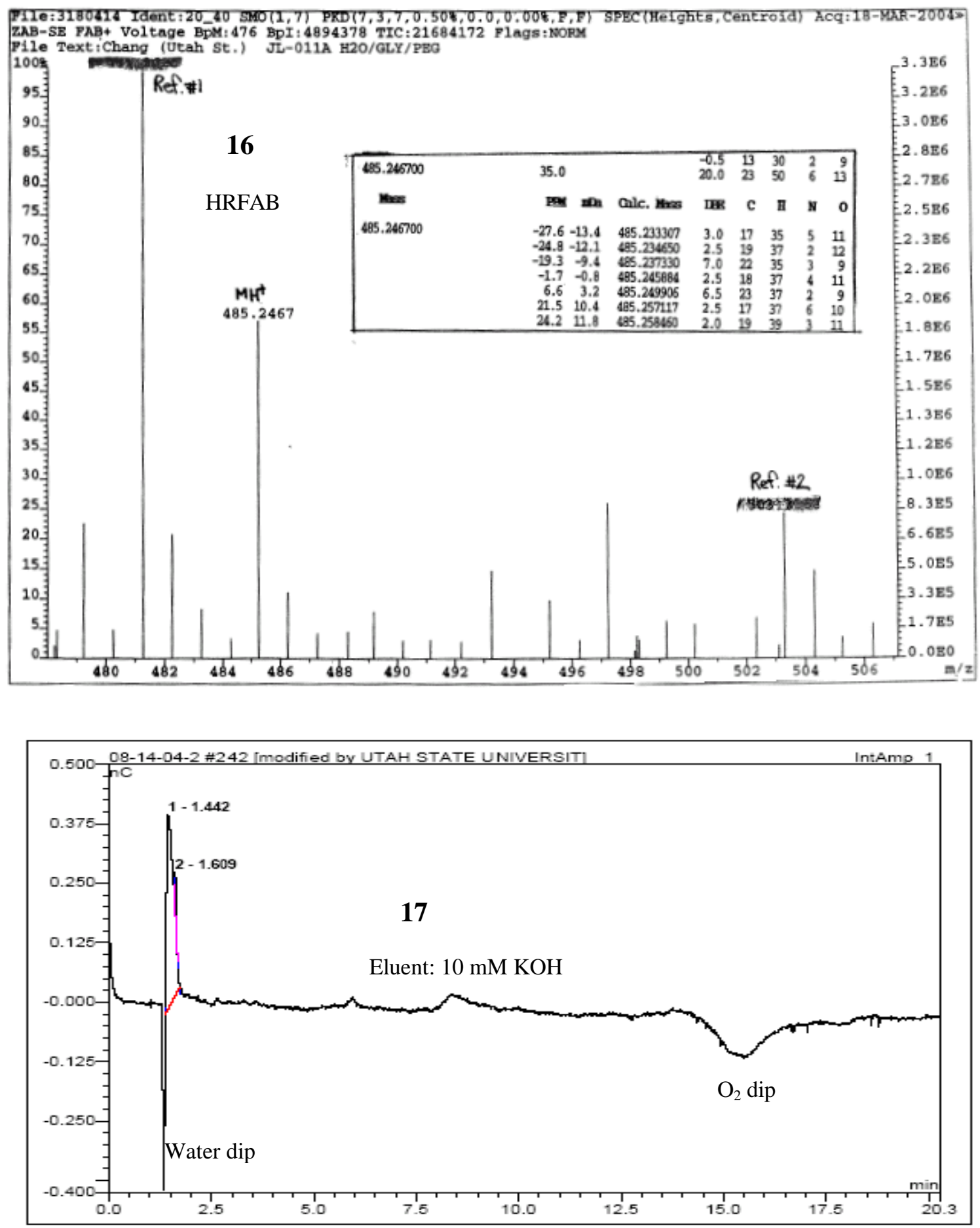

\begin{tabular}{|c|c|c|c|c|c|c|c|}
\hline No. & $\begin{array}{c}\text { Ret.Time } \\
\text { min }\end{array}$ & Peak Name & $\begin{array}{c}\text { Height } \\
\text { nC }\end{array}$ & $\begin{array}{c}\text { Area } \\
\mathrm{nC}^{*} \mathrm{~min}\end{array}$ & $\begin{array}{c}\text { Rel.Area } \\
\%\end{array}$ & $\begin{array}{c}\text { Amount } \\
\text { ppm }\end{array}$ & Type \\
\hline 1 & 1.44 & n.a. & 0.408 & 0.082 & 95.20 & n.a. & BMB* \\
\hline 2 & 1.61 & n.a. & 0.069 & 0.004 & 4.80 & n.a. & $\mathrm{Rd}$ \\
\hline
\end{tabular}



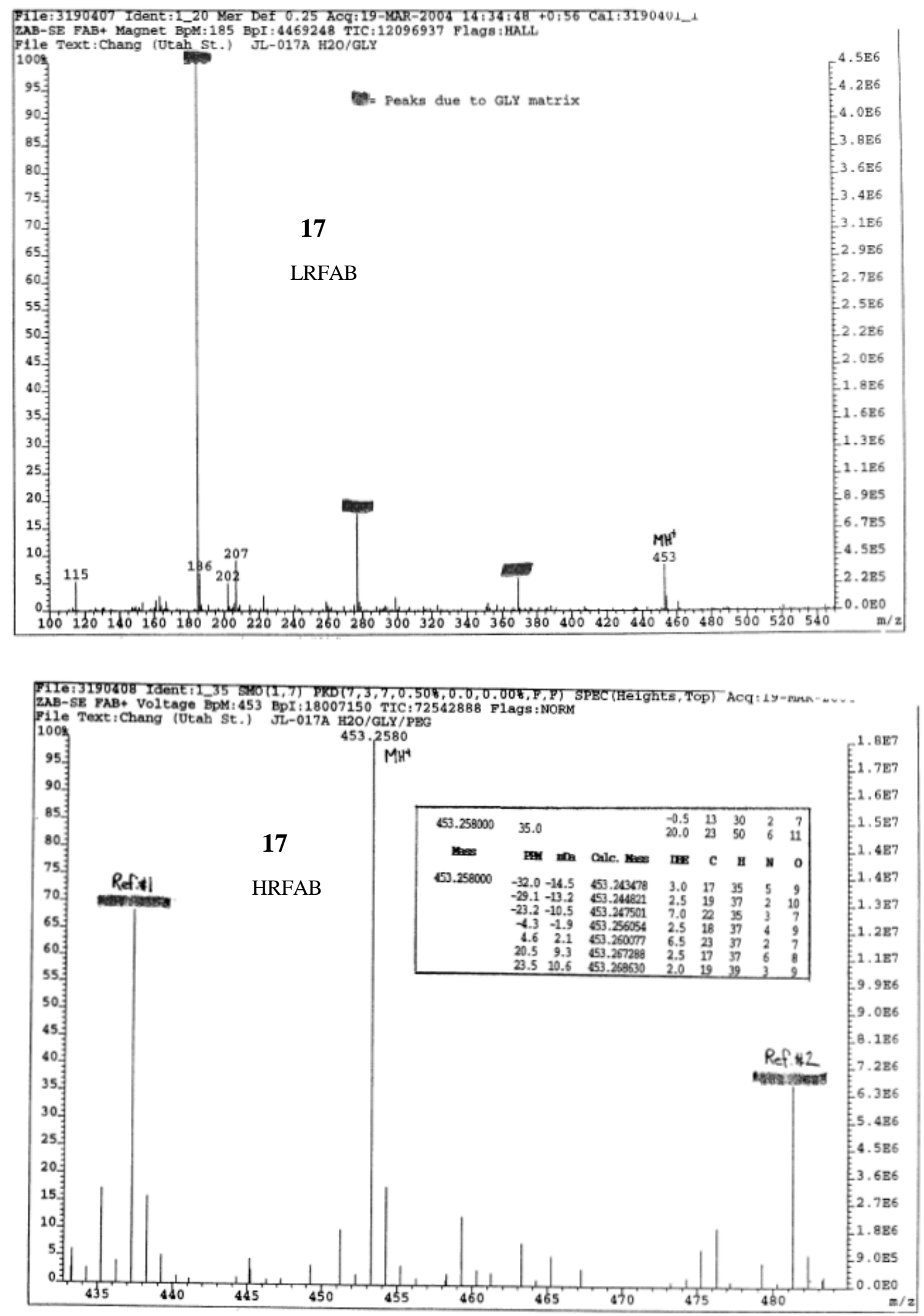


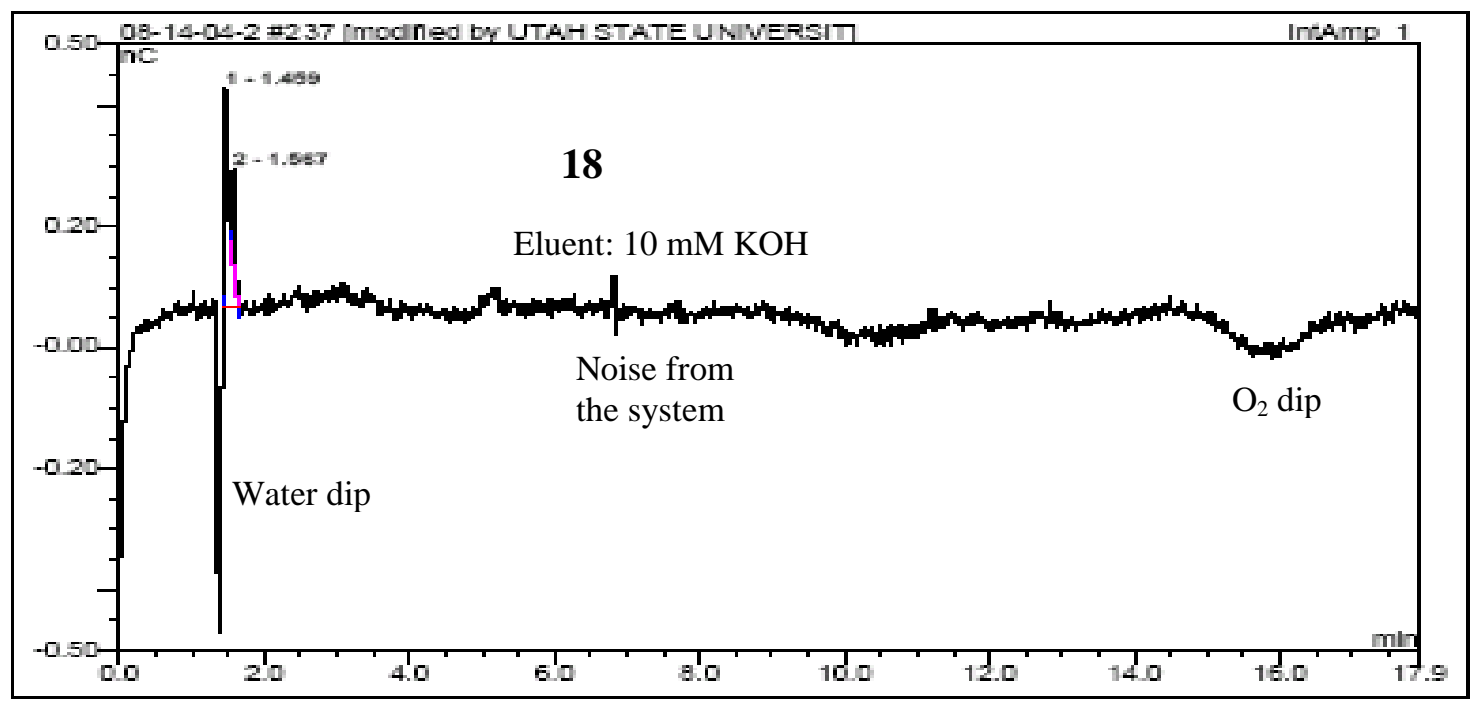

\begin{tabular}{|c|c|c|c|c|c|c|c|}
\hline No. & $\begin{array}{c}\text { Ret.TIme } \\
\text { m In }\end{array}$ & Peak Name & $\begin{array}{l}\text { Helght } \\
\text { nc }\end{array}$ & $\begin{array}{c}\text { Area } \\
\text { nctmin }\end{array}$ & $\begin{array}{c}\text { Rel.Area } \\
\% 6\end{array}$ & $\begin{array}{c}\text { Amount } \\
\text { ppm }\end{array}$ & Type \\
\hline
\end{tabular}

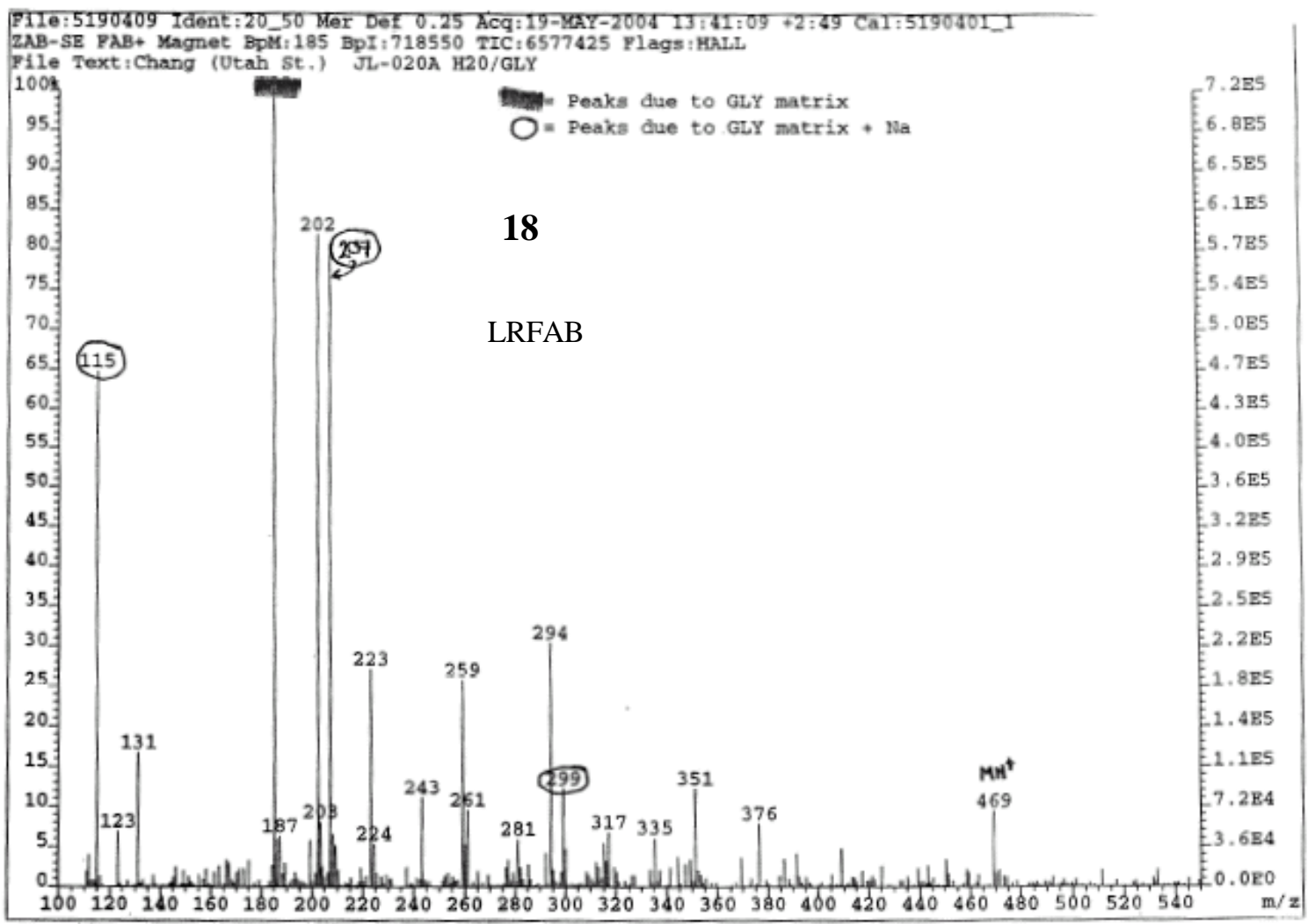




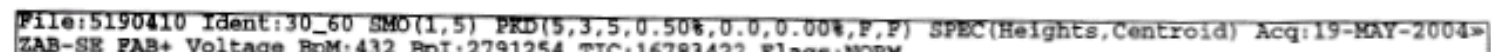
ZAB-SE PAB+ Voltage BpM:432 BpI:2791254 TIC:16783422 Flags : Nopo

Pile Text: Chang (Utah St.) JL-020A H20/GLY/PEG

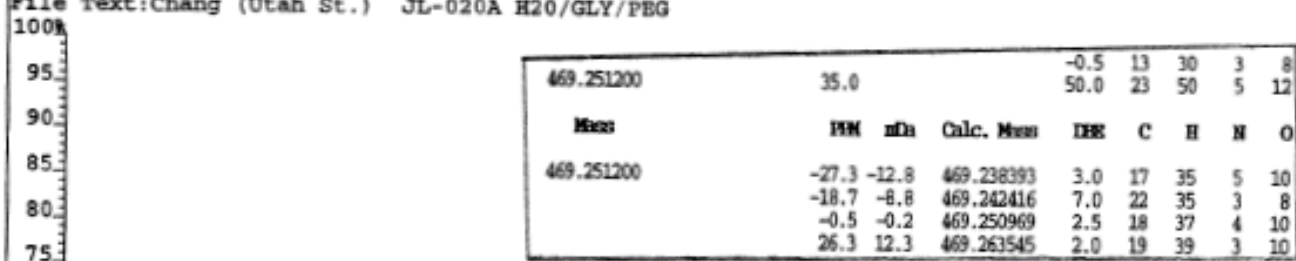

$1.0 \mathrm{E} 6$

9. $9 \mathrm{BS}$

9.385

8. $8 \mathrm{E5}$

8. $2 \mathrm{ES}$

7.785

7.1Es

$6.6 \mathrm{~B} 5$

6. 085

$5.5 E 5$

$4.9 \mathrm{~B} 5$

HRFAB

469.2512

Ref. $* 2$

4.485

353

30 격

25

$20 \frac{3}{3}$

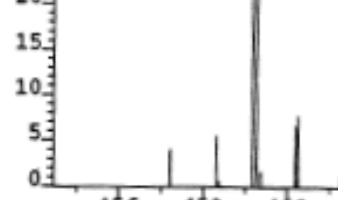

$\begin{array}{lll}456 & 458\end{array}$

$\begin{array}{lll}464 & 466 & 468\end{array}$
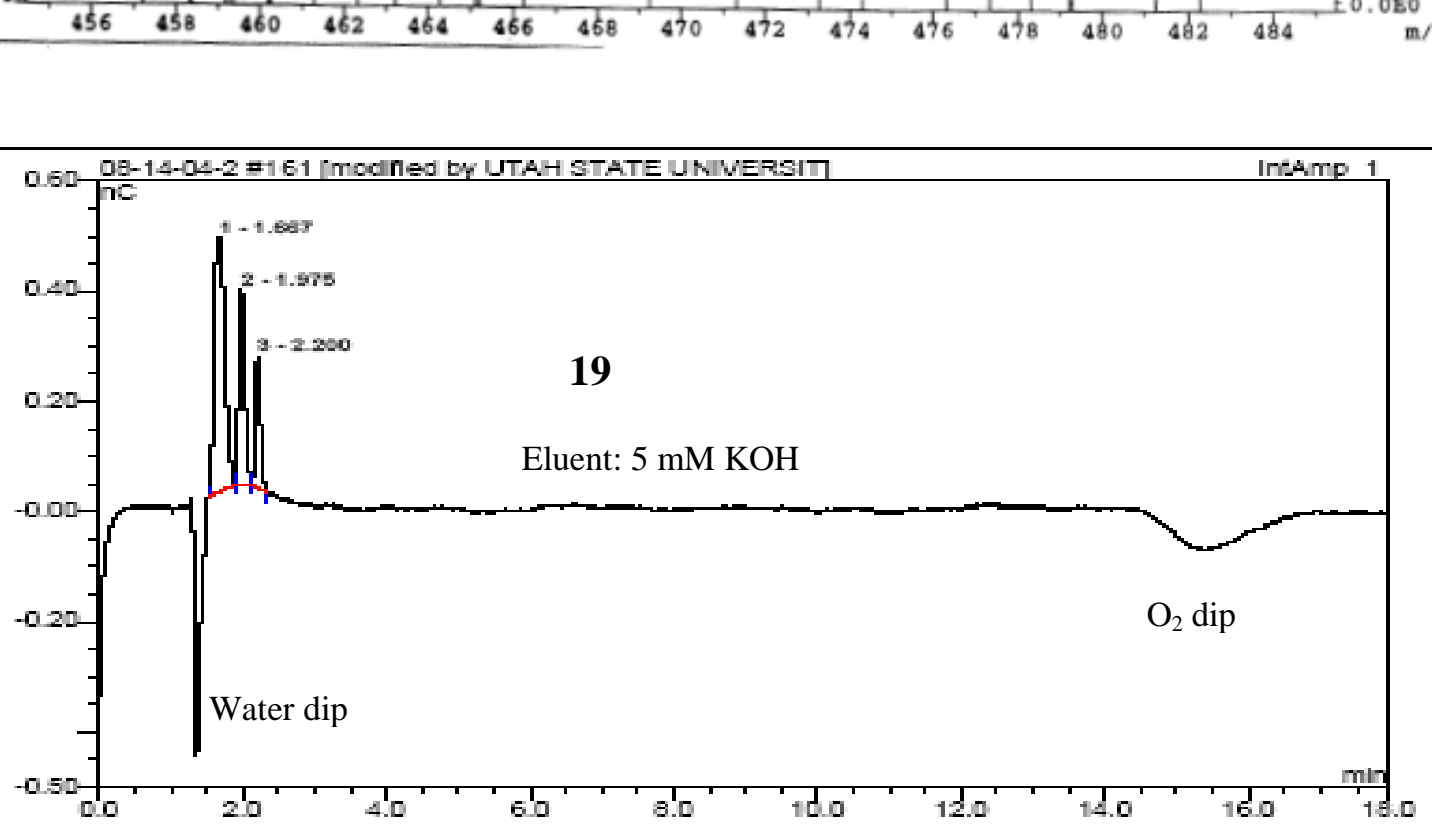

\begin{tabular}{|c|c|c|c|c|c|c|c|}
\hline No. & $\begin{array}{c}\operatorname{Ret} \operatorname{TIm} \theta \\
\mathrm{m} / \mathrm{n}\end{array}$ & Peak Name & $\begin{array}{l}\text { Helght } \\
\text { nc }\end{array}$ & $\begin{array}{c}\text { Area } \\
\mathrm{nc}^{+} \mathrm{m} / \mathrm{n}\end{array}$ & $\begin{array}{c}\text { Rel.Area } \\
\%\end{array}$ & $\begin{array}{c}\text { Amount } \\
\text { ppm }\end{array}$ & Type \\
\hline 2 & 198 & n.a. & 0.354 & 0.034 & 25.02 & n.a. & bMo \\
\hline Total: & & & 1.055 & 0.134 & 100.00 & 0.000 & \\
\hline
\end{tabular}



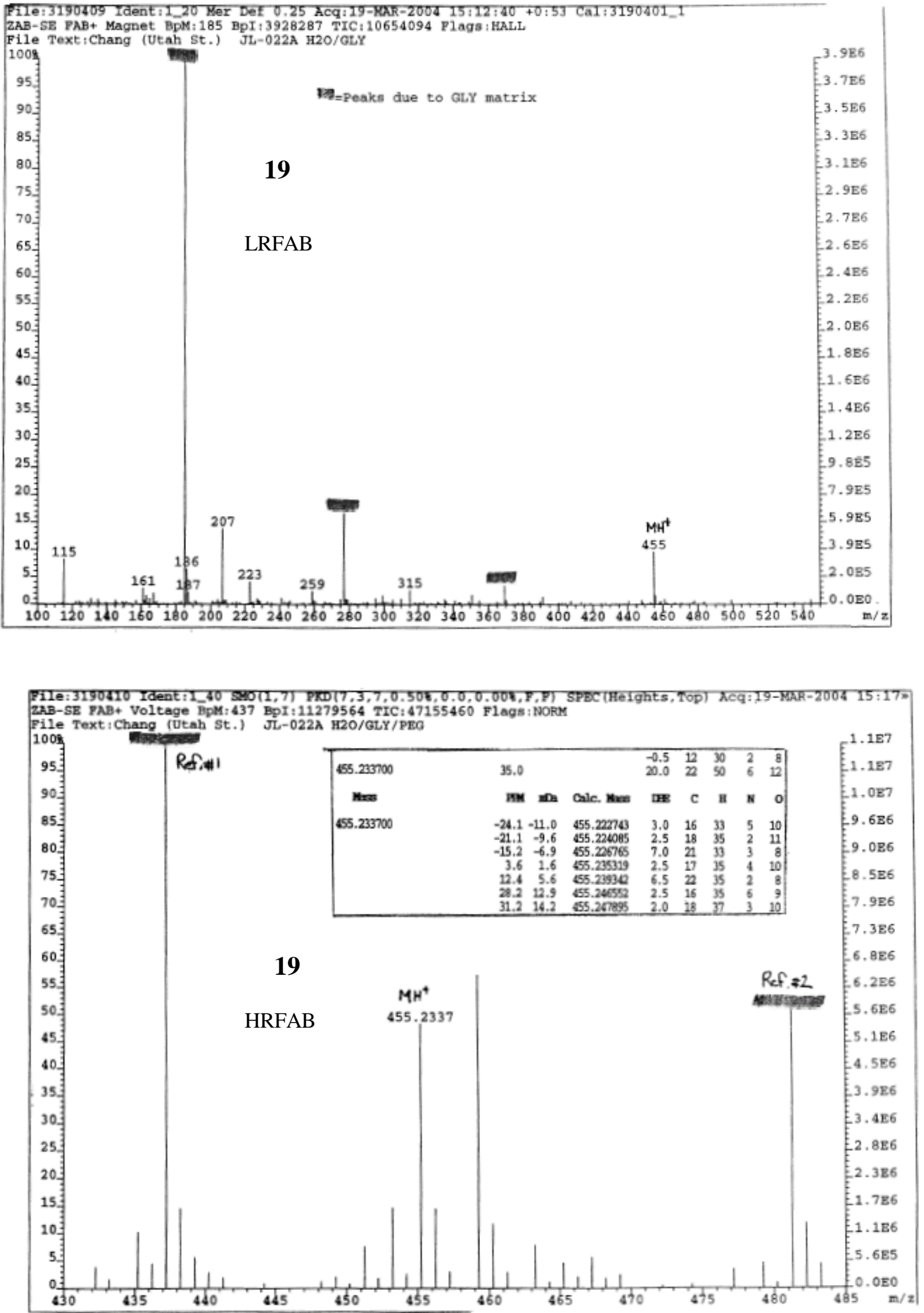


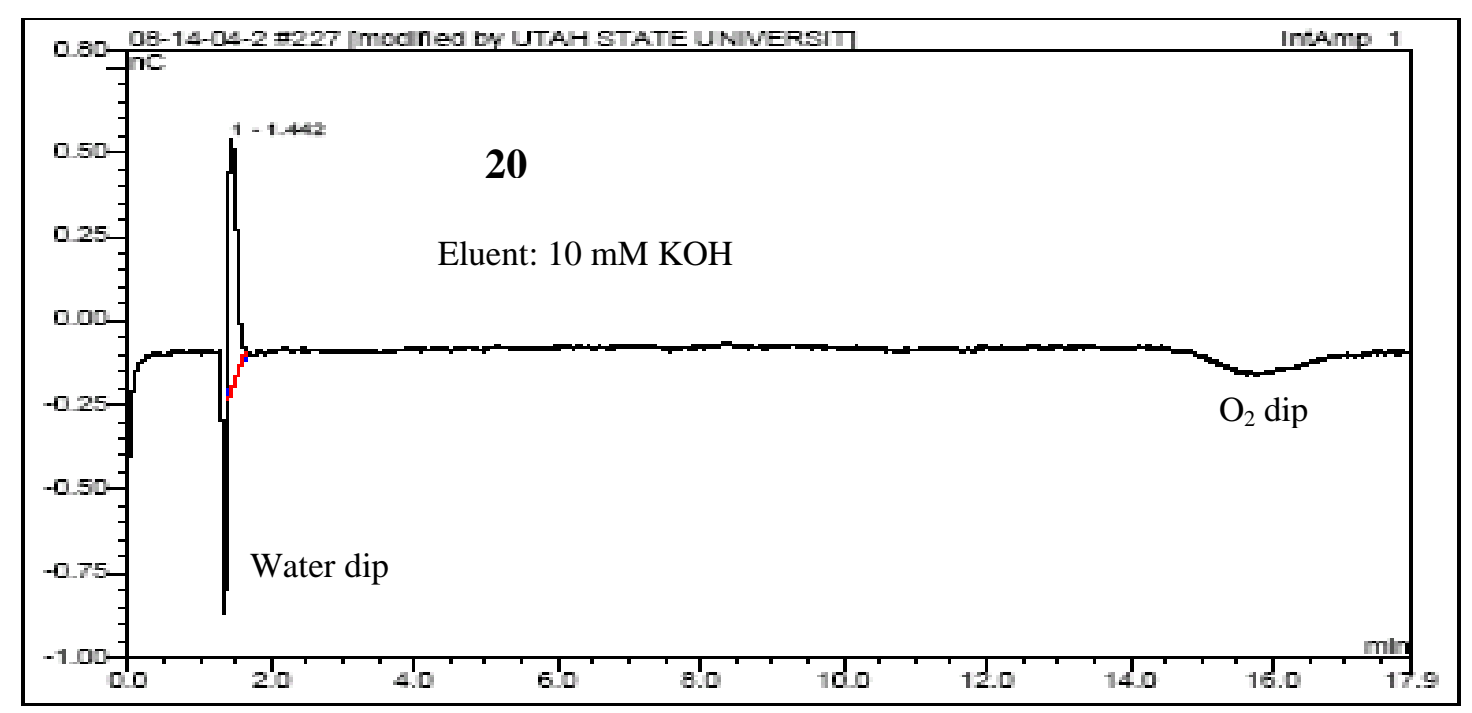

\begin{tabular}{|c|c|c|c|c|c|c|c|}
\hline No. & $\begin{array}{c}\text { Ret.TIme } \\
\mathrm{m} / \mathrm{n}\end{array}$ & Peak Name & $\begin{array}{c}\text { Helght } \\
\text { nc }\end{array}$ & $\begin{array}{c}\text { Area } \\
\text { nctmin }\end{array}$ & $\begin{array}{c}\text { Rel.Area } \\
\%\end{array}$ & $\begin{array}{c}\text { Amount } \\
\text { pom }\end{array}$ & Type \\
\hline 1 & $1 \leqslant 4$ & n.a. & 0.741 & 0.092 & 100.00 & n.a. & BME \\
\hline Total: & & & 0.741 & 0.092 & 100.00 & 0.000 & \\
\hline
\end{tabular}

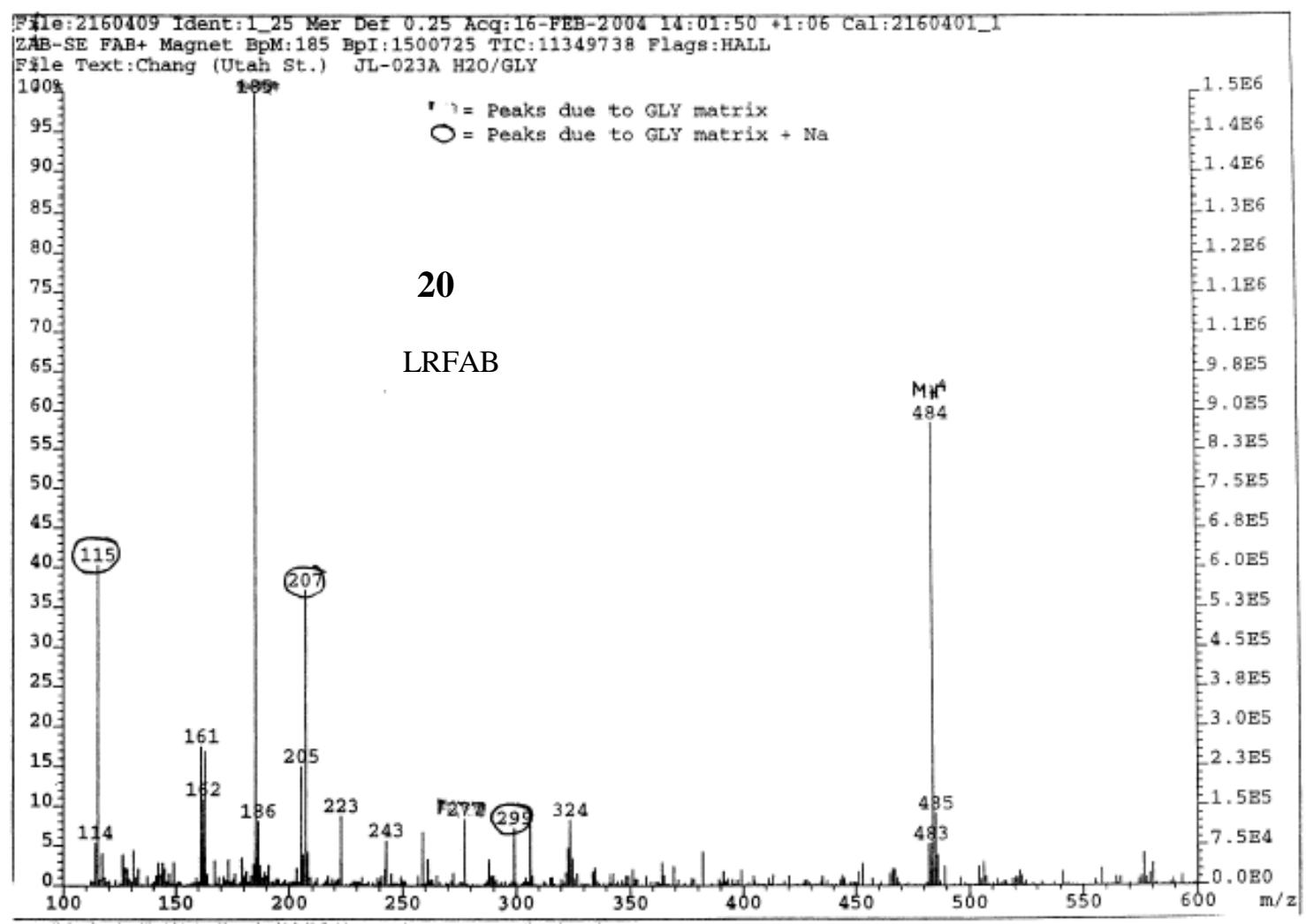



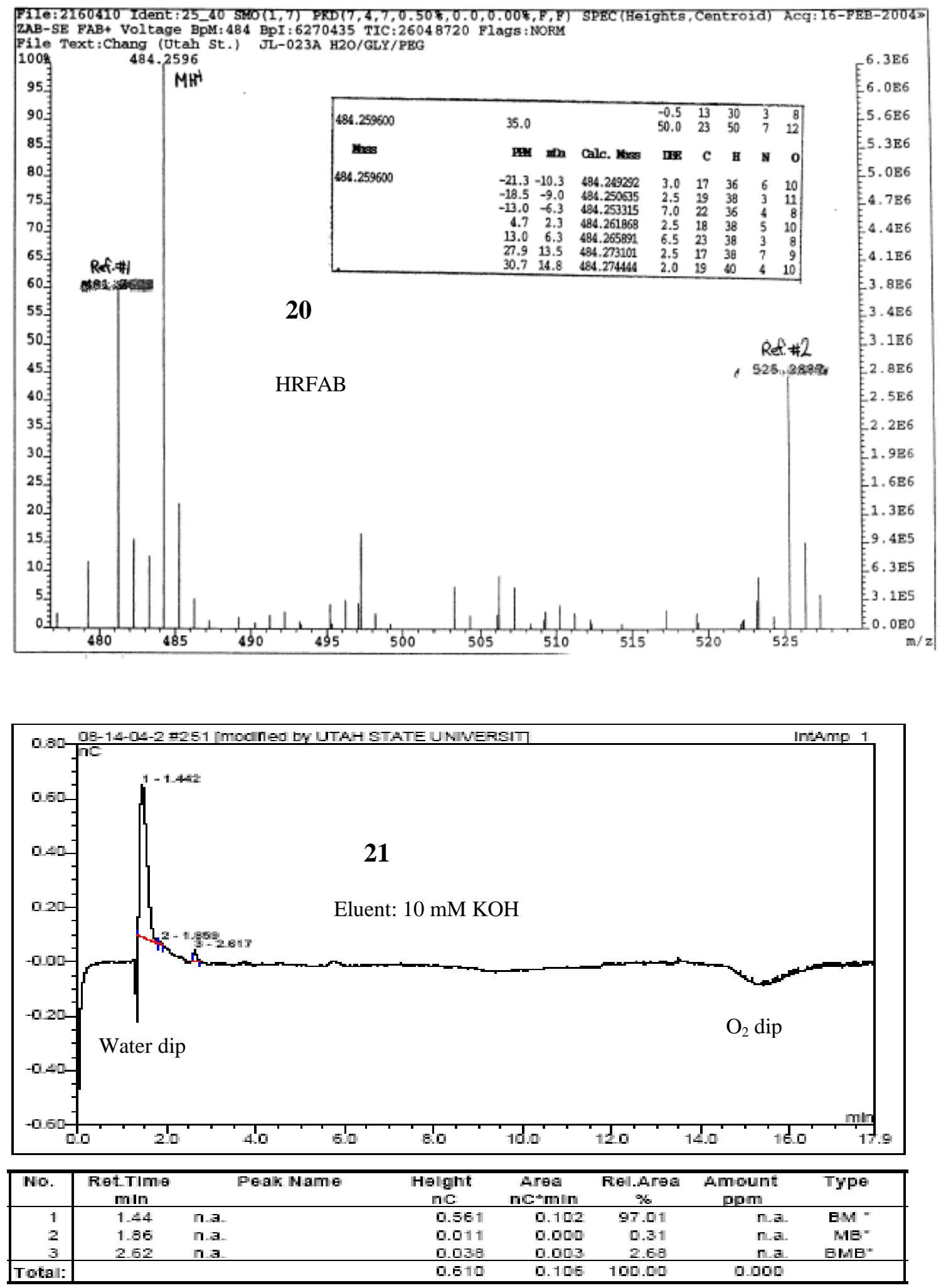

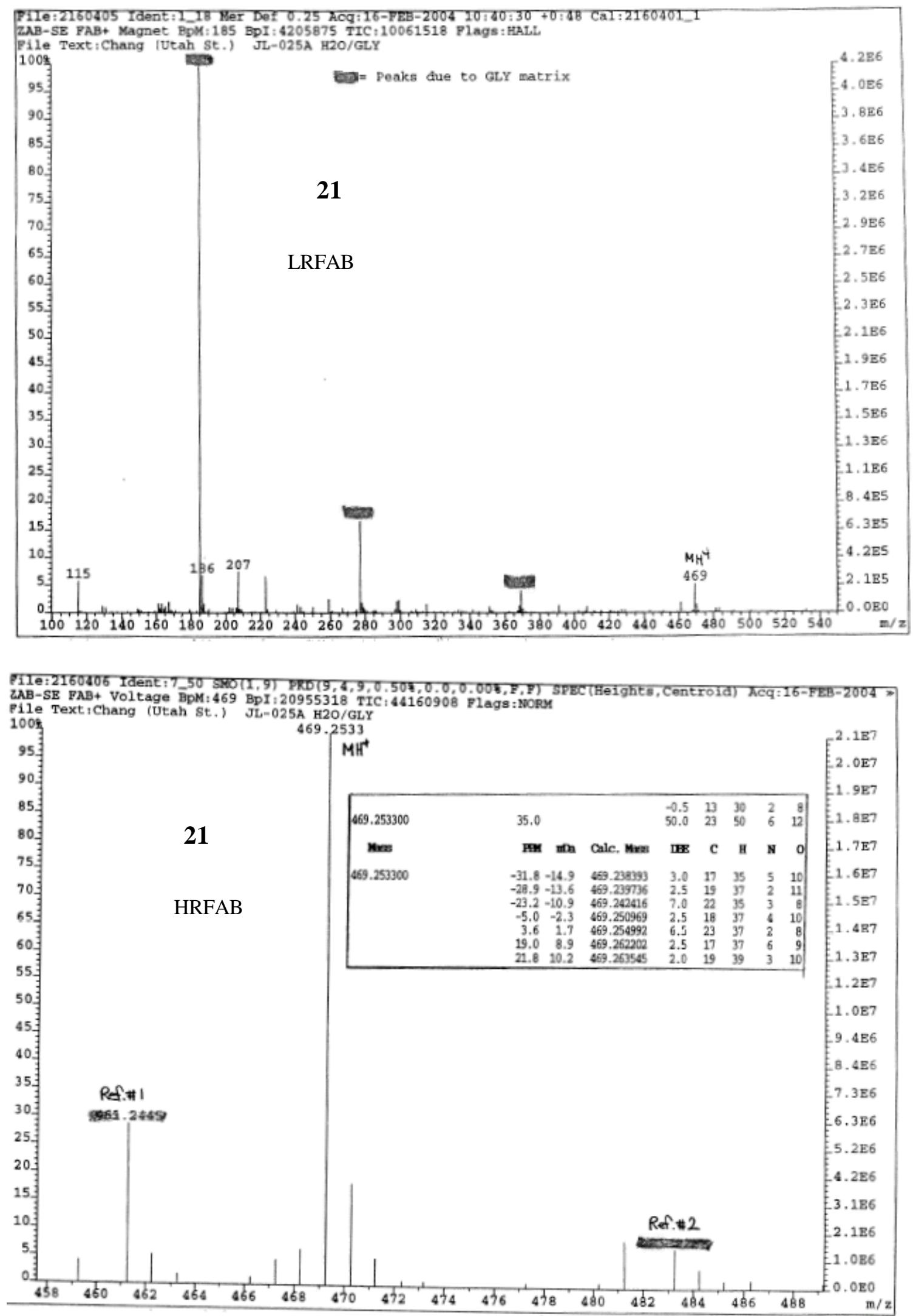

S11 

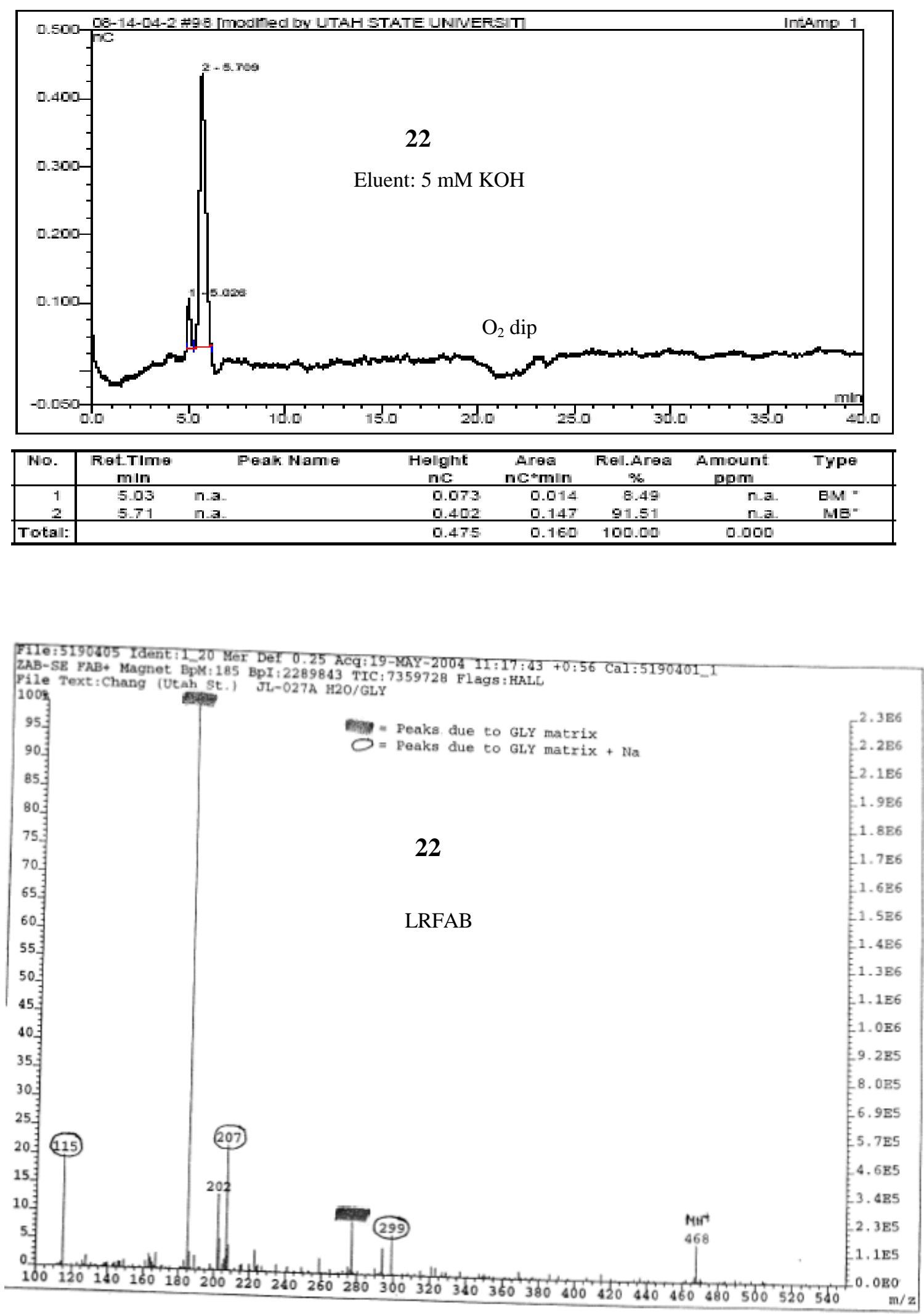

S12 

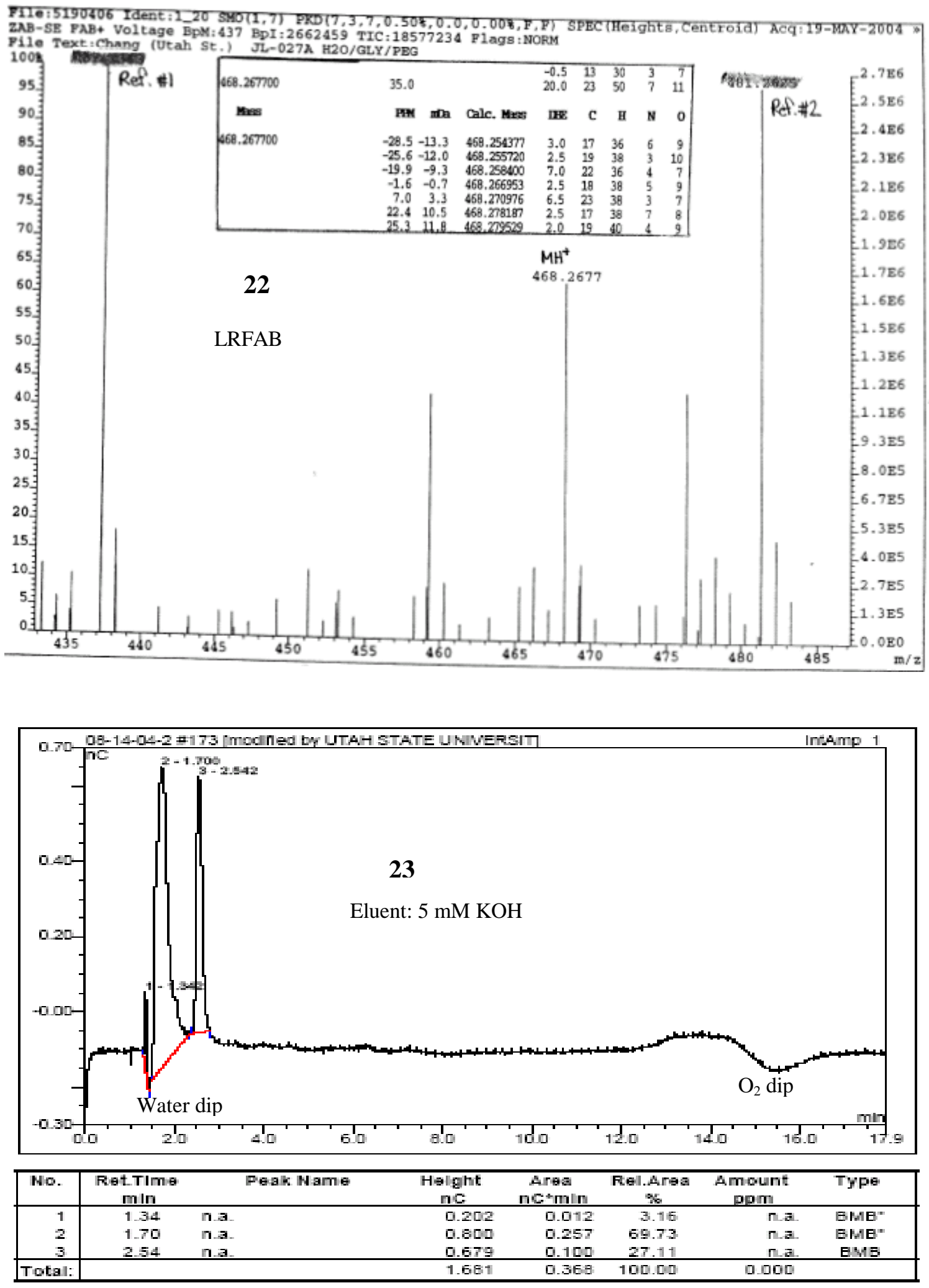

S13 

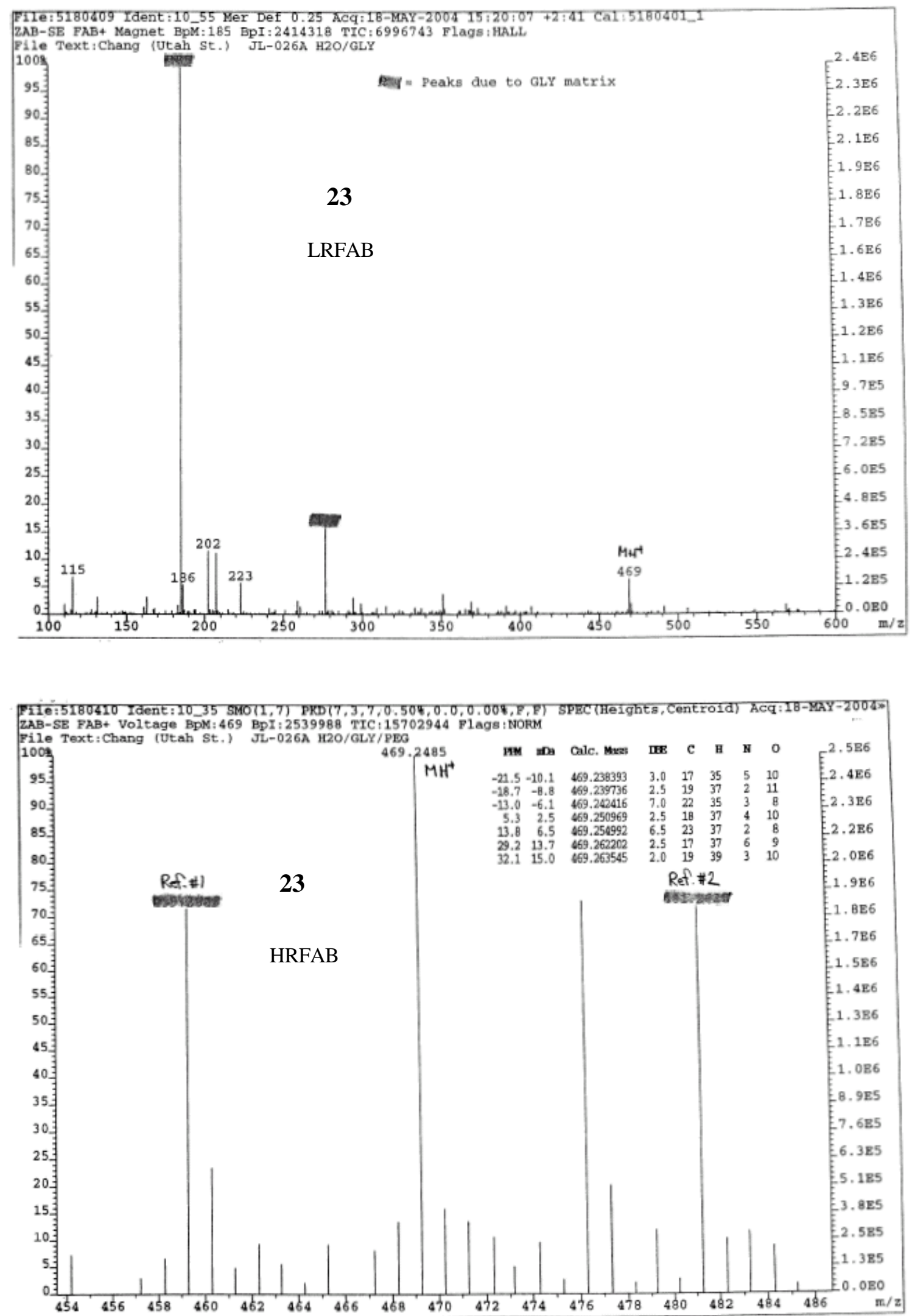

S14 

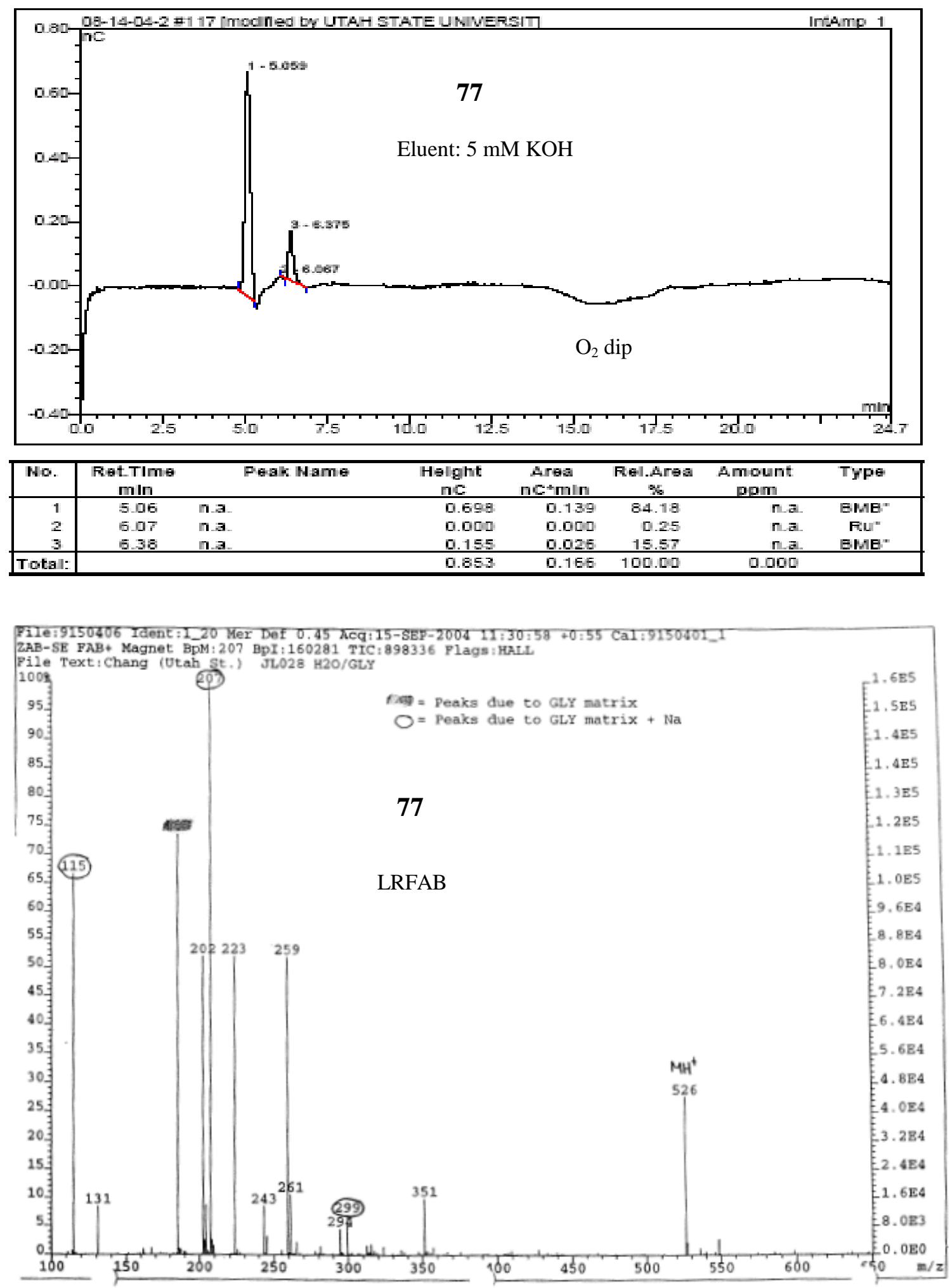

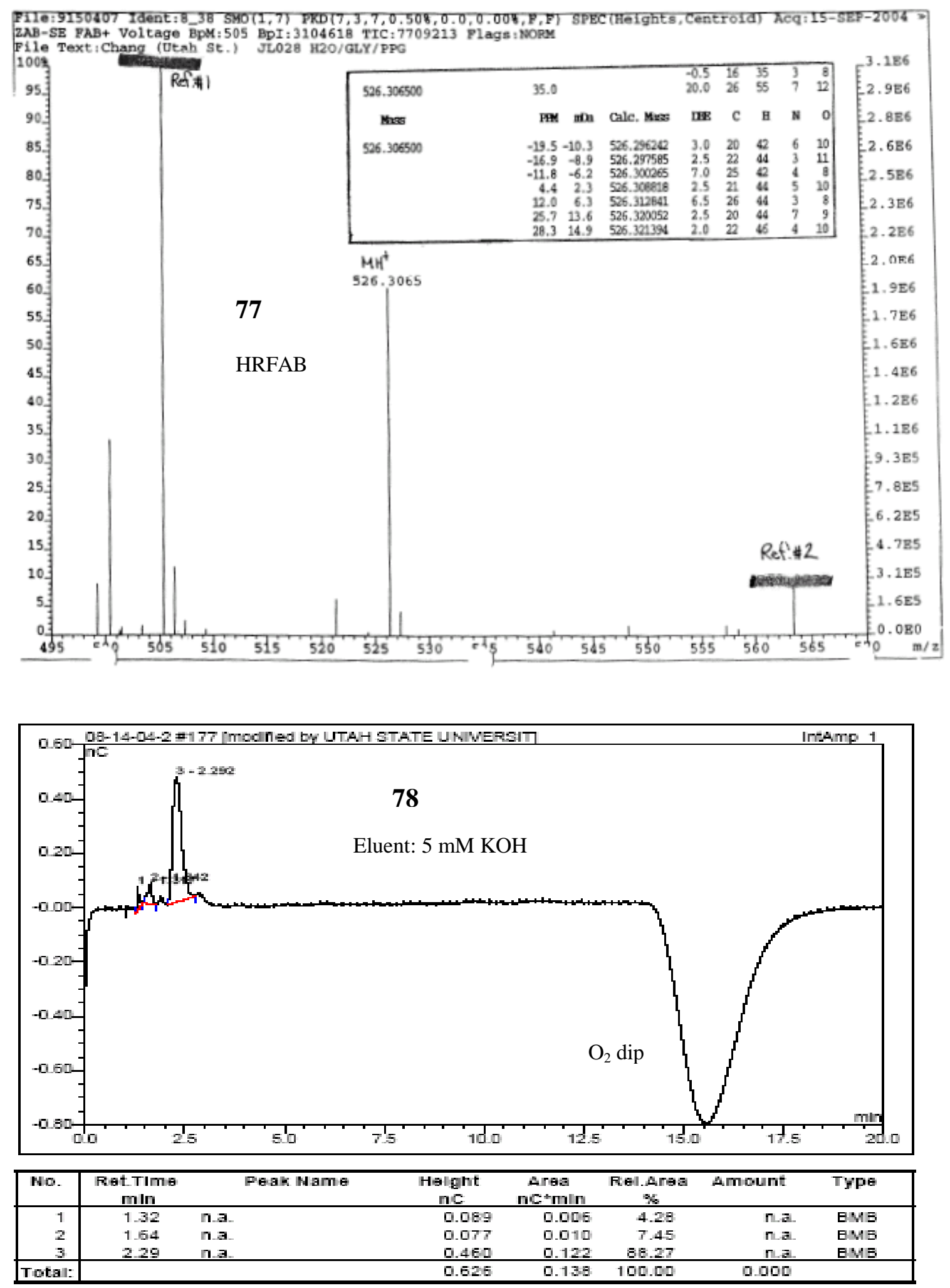

S16 

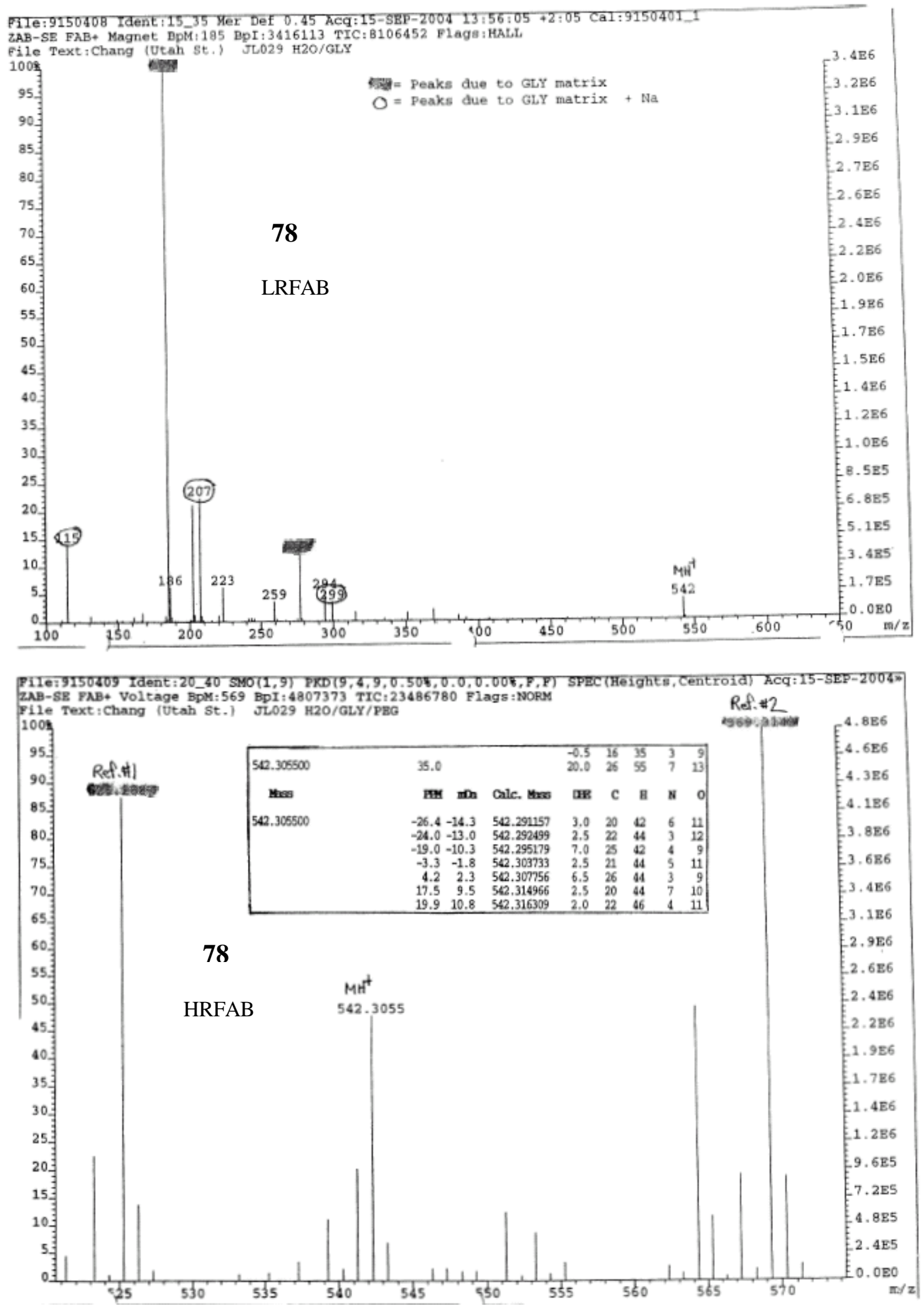

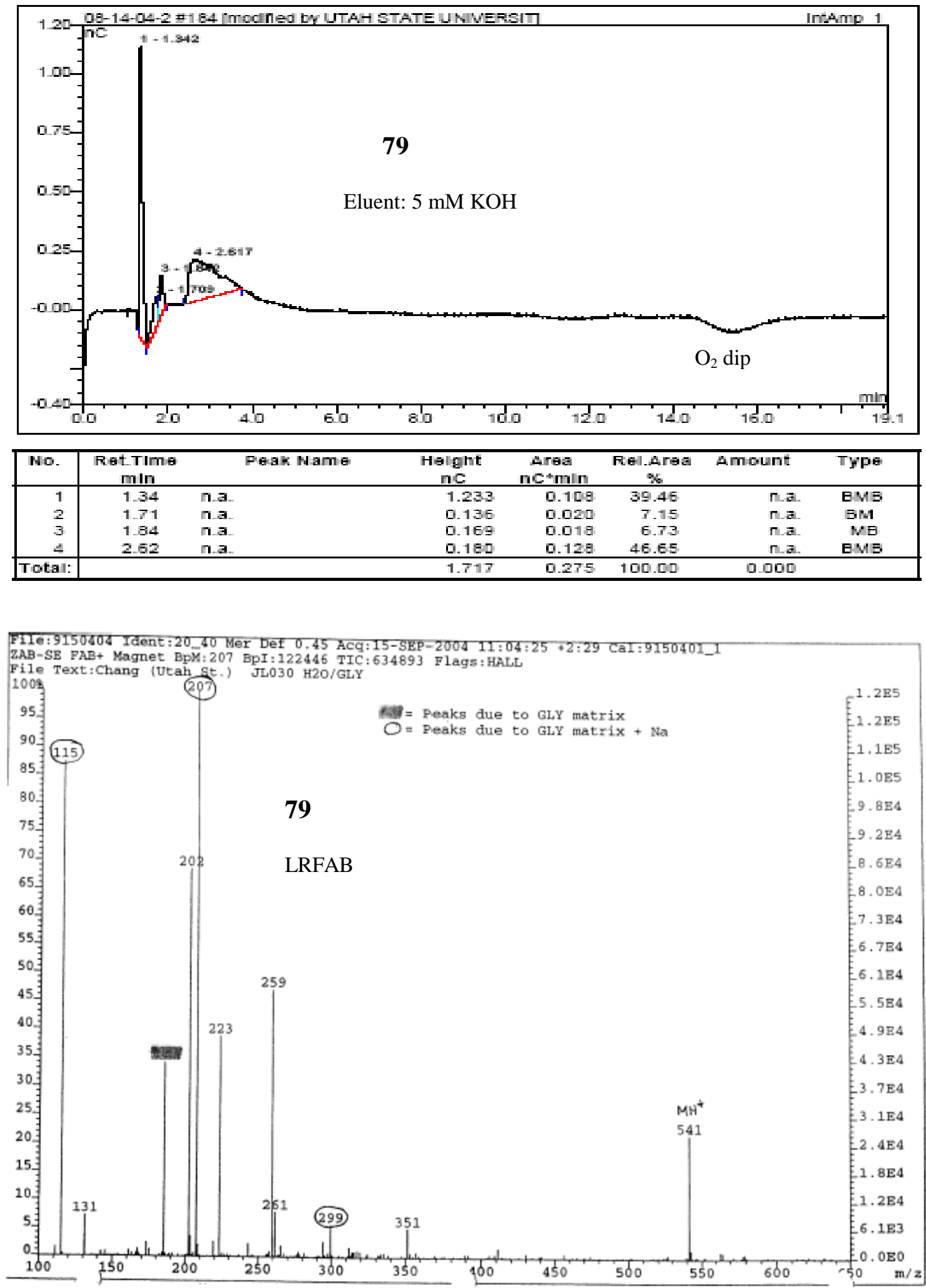

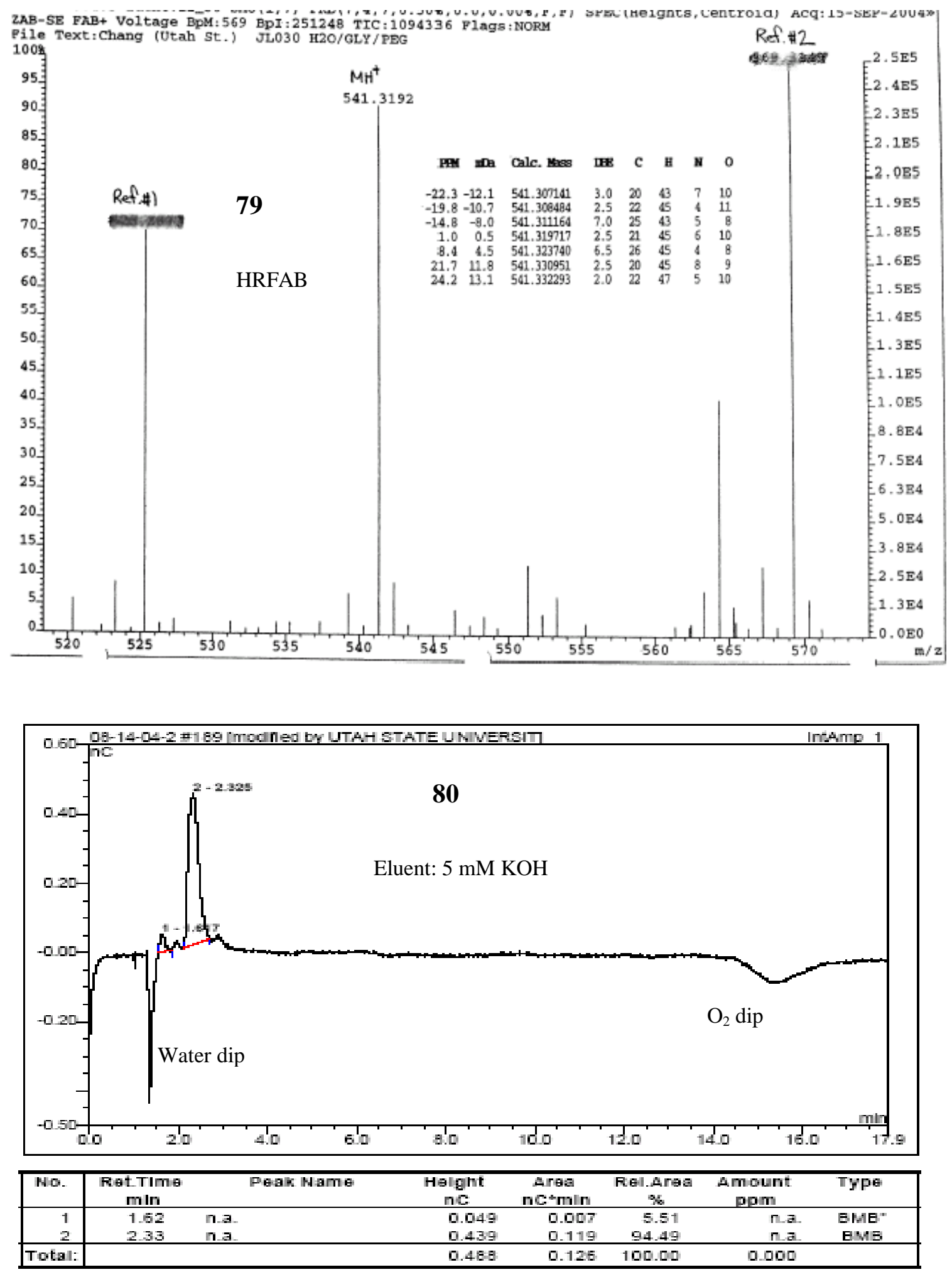

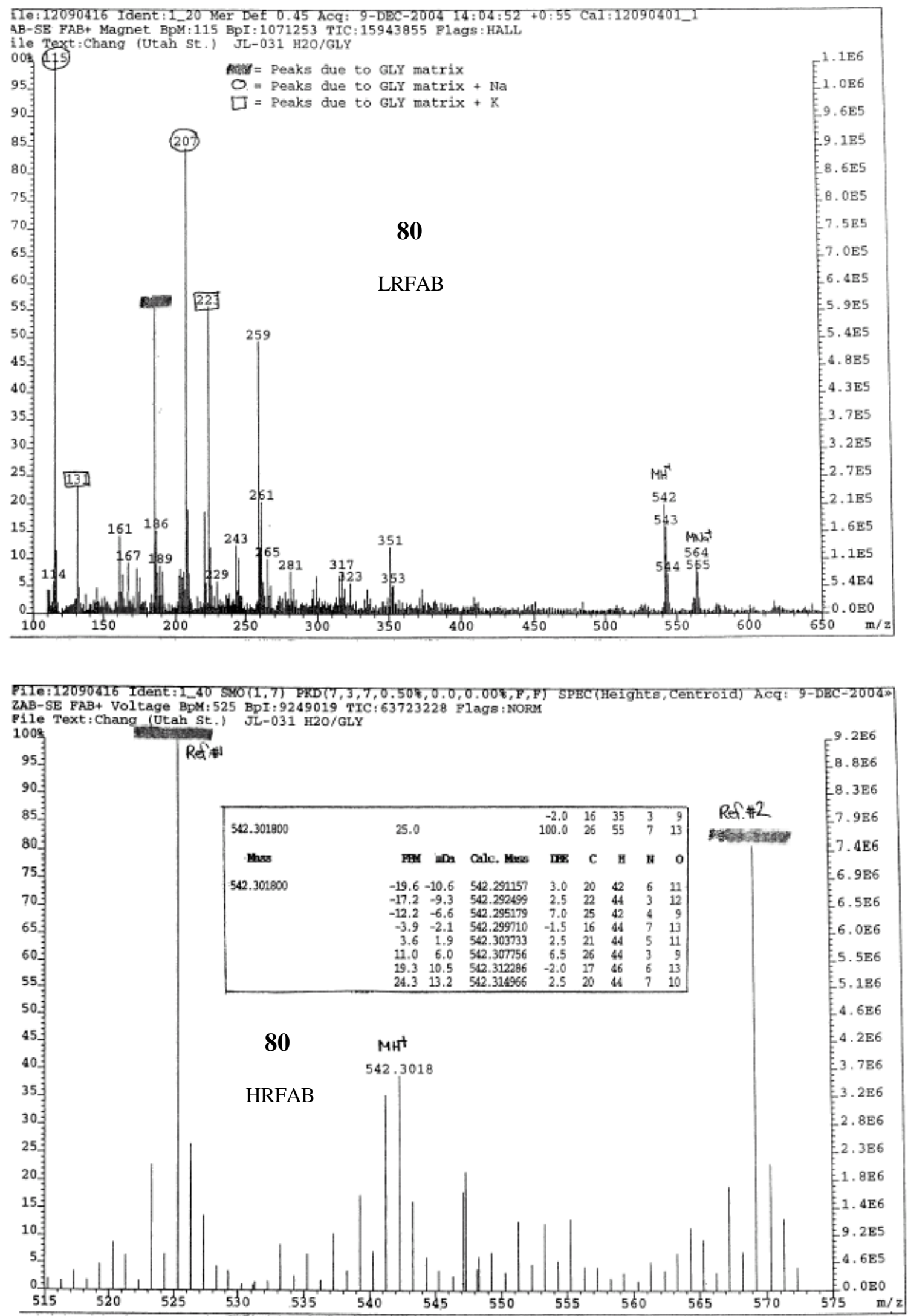

S20 

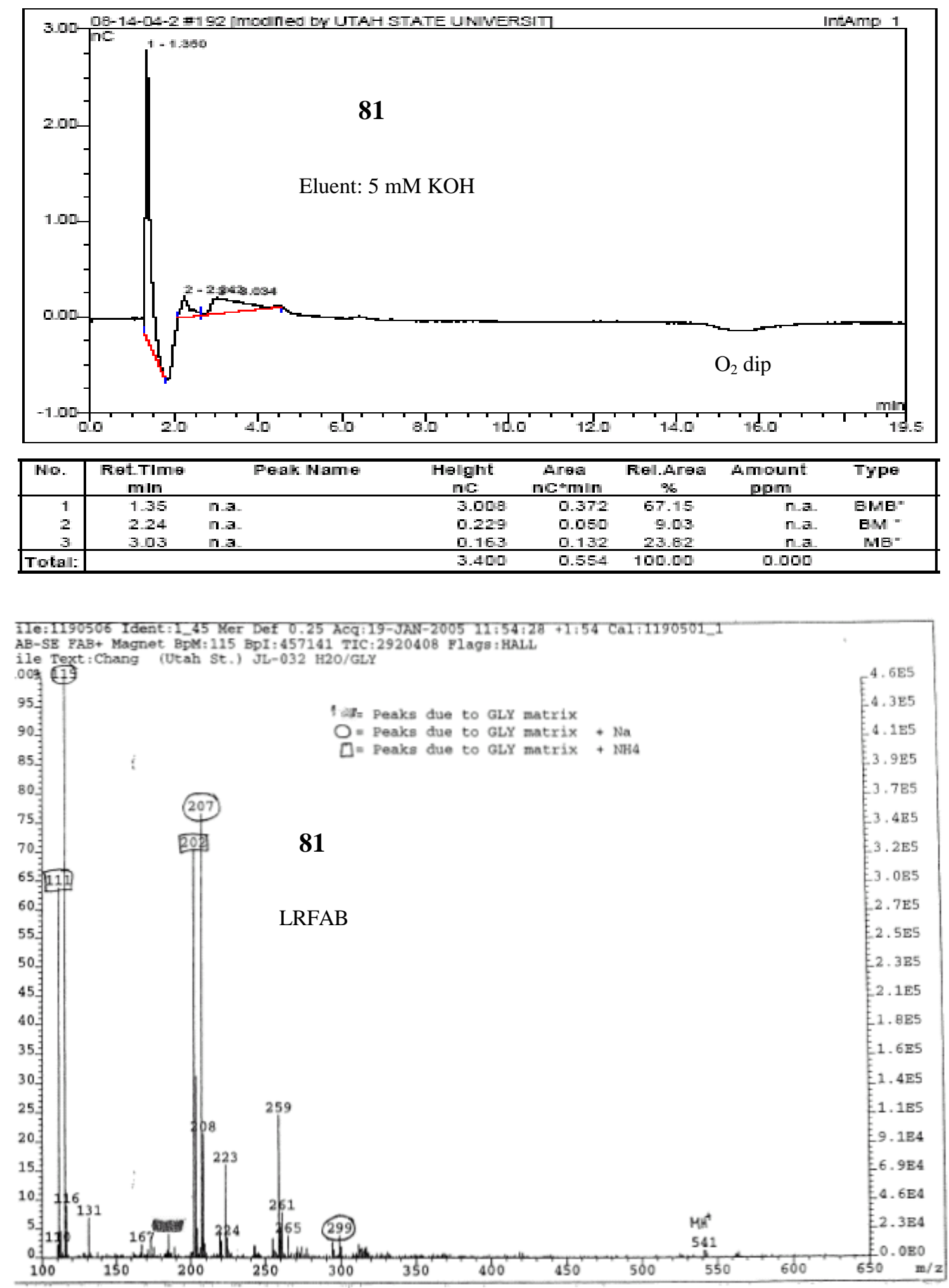

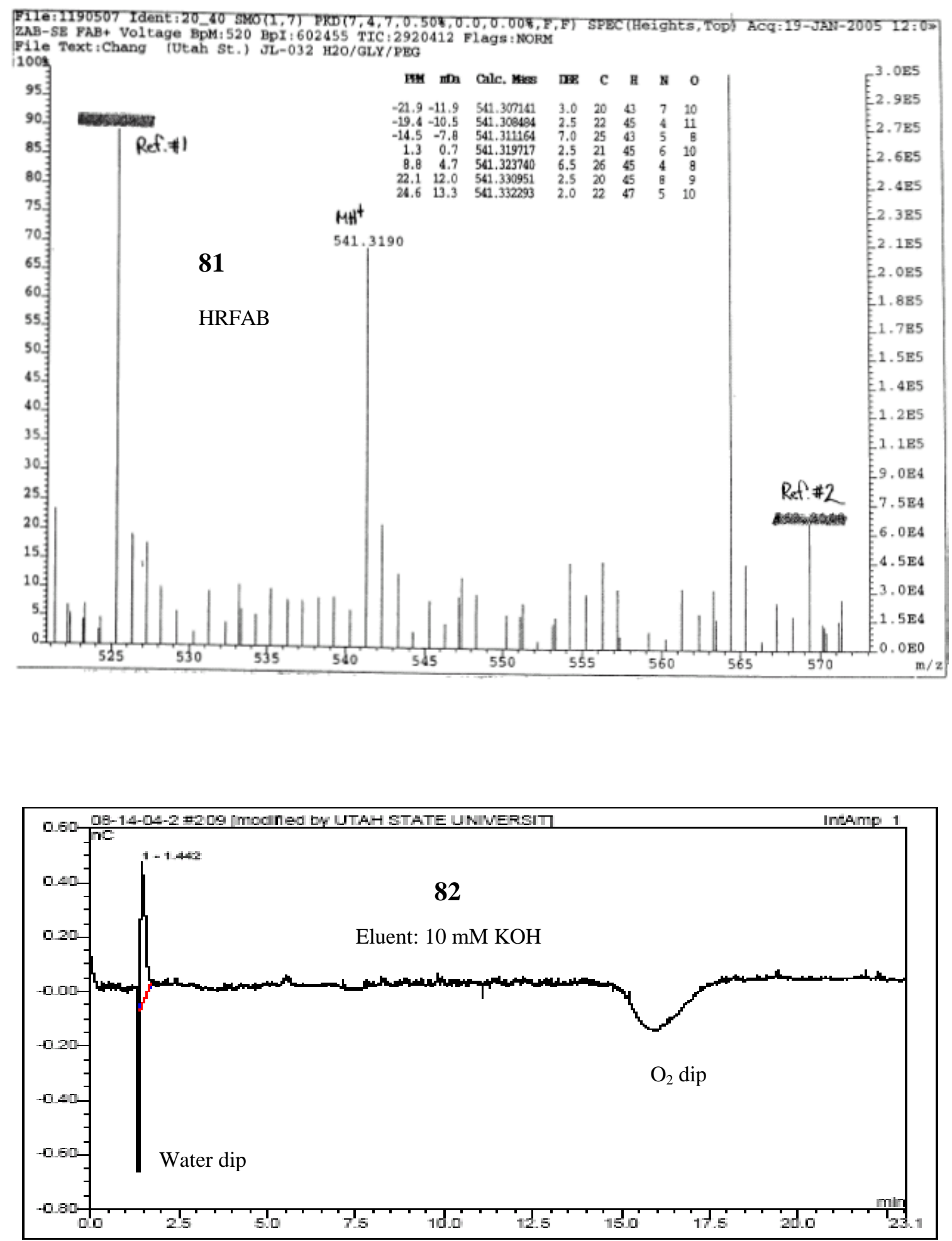

\begin{tabular}{|c|c|c|c|c|c|c|c|}
\hline No. & $\begin{array}{c}\text { Ret.TIme } \\
\text { min }\end{array}$ & Peak Name & $\begin{array}{c}\text { Helght } \\
\text { nc }\end{array}$ & $\begin{array}{c}\text { Area } \\
\text { nctmin }\end{array}$ & $\begin{array}{c}\text { Rel.Area } \\
\%_{6}\end{array}$ & $\begin{array}{c}\text { Amount } \\
\text { pom }\end{array}$ & Type \\
\hline 1 & $1 \leqslant 4$ & n.3. & 0.531 & 0.075 & 100.00 & n.a. & BME \\
\hline Total: & & & 0.531 & 0.075 & 100.00 & 0.000 & \\
\hline
\end{tabular}



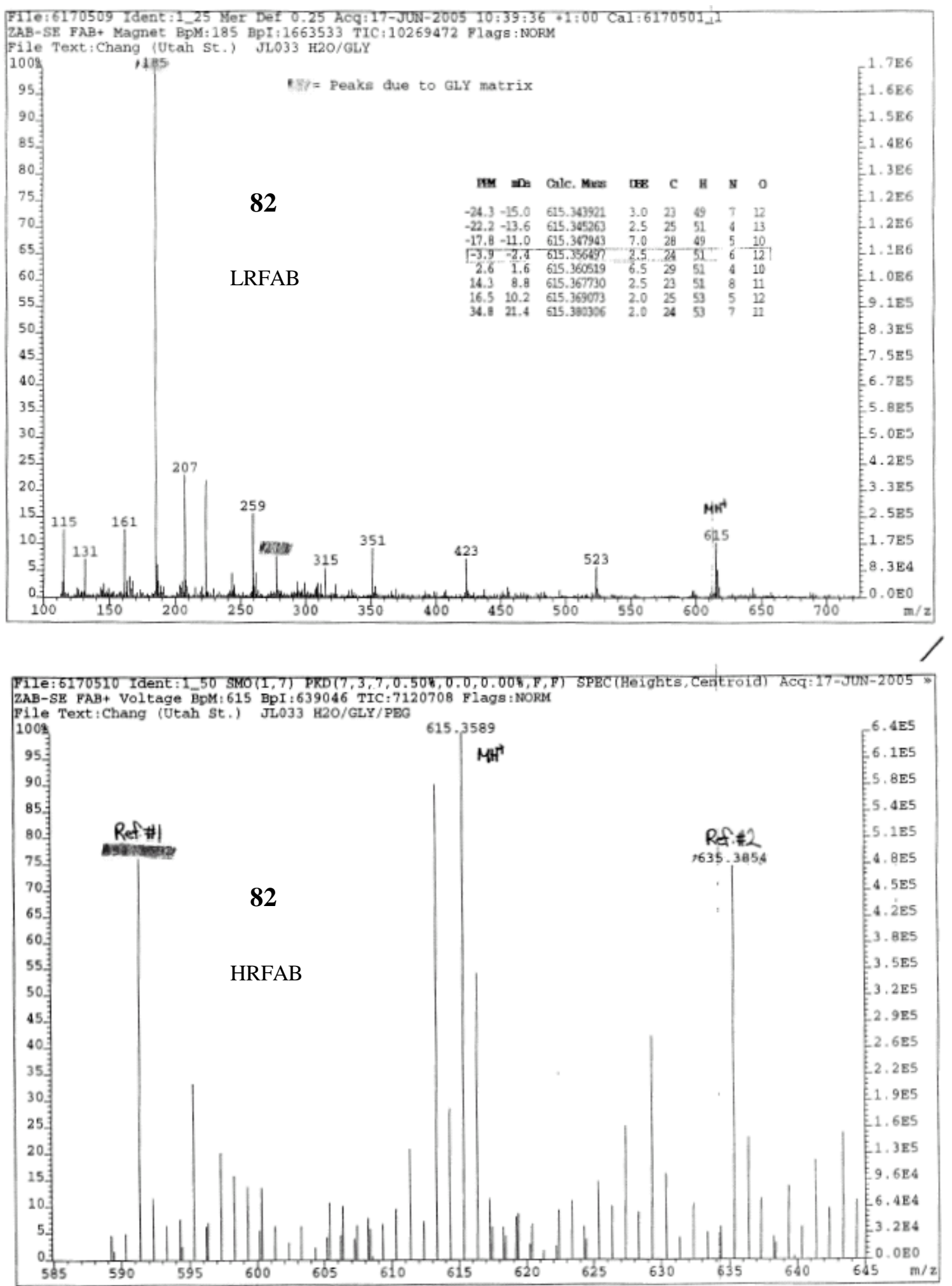

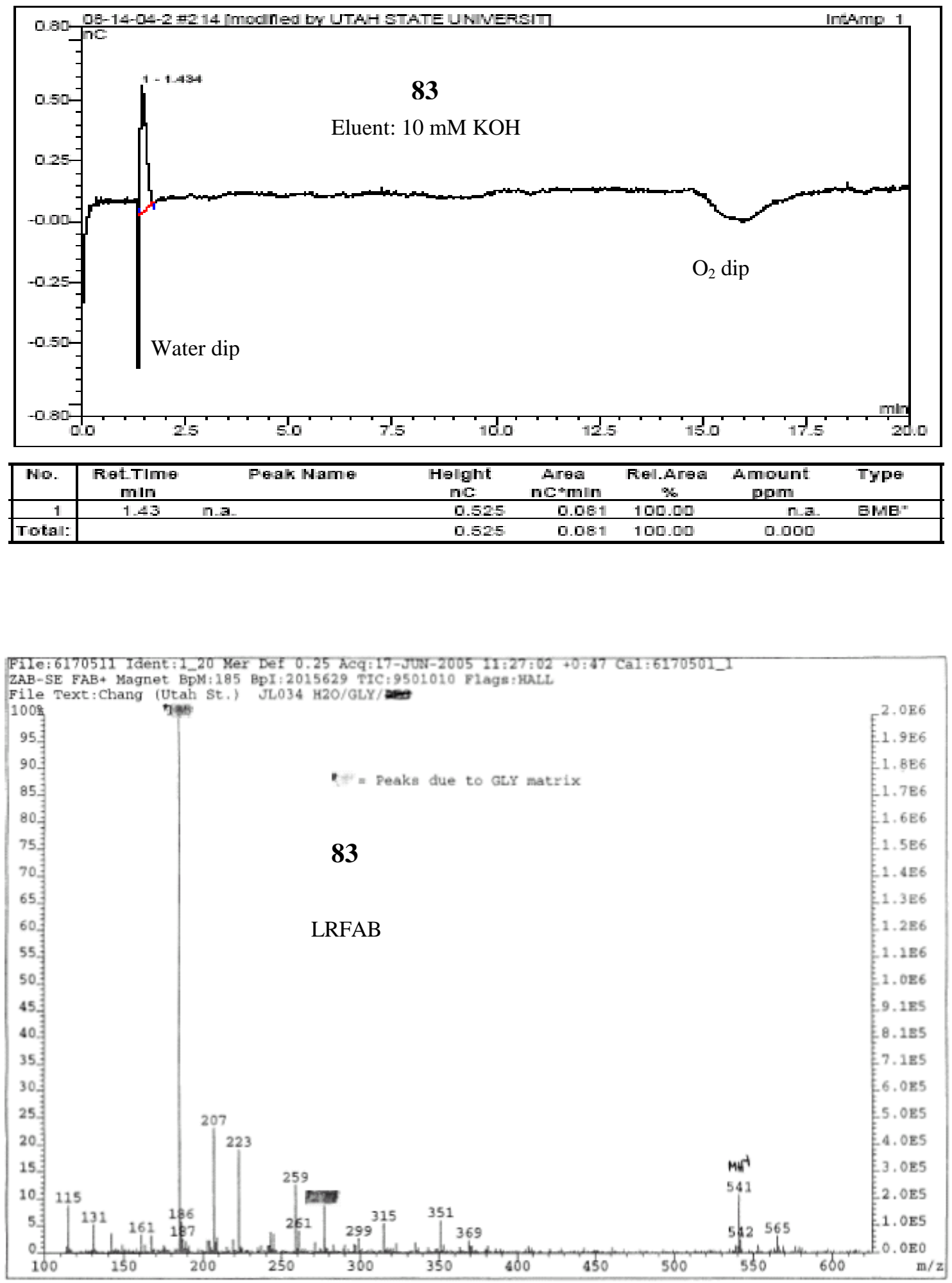


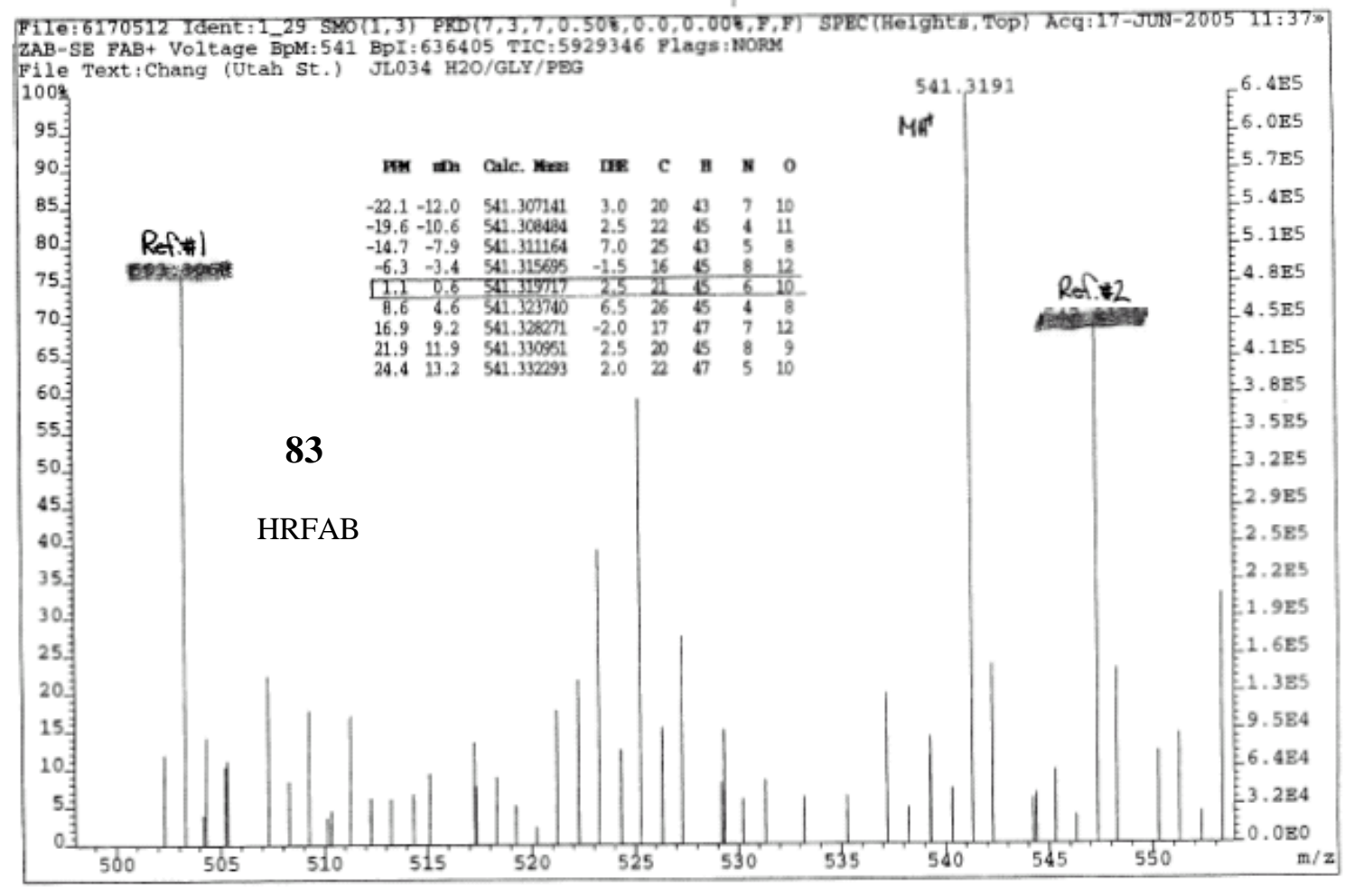

